\title{
Prevalência de risco de sarcopenia e obesidade sarcopênica entre idosos não-institucionalizados do interior do Ceará
}

\author{
Prevalence of risk of sarcopenia and sarcopenic obesity among non-institutionalized elderly in the \\ interior of Ceará
}

DOI: $10.37111 /$ braspenj.2020354010

Orianny Nágela Batista Santos ${ }^{1}$

Bruna Yhang da Costa Silva²

Thais Ariele Lima Chaves'

Ozianne Kelly Vidal Oliveira ${ }^{3}$

Jane Karine da Silva ${ }^{3}$

Samanta Naje Rodrigues de Castro $^{3}$

\section{Unitermos:}

Sarcopenia. Obesidade. Envelhecimento. Qualidade de vida. Idoso fragilizado.

\section{Keywords:}

Sarcopenia. Obesity. Aging. Quality of life. Frail Elderly.

\section{Endereço para correspondência:}

Bruna Yhang da Costa Silva

Rua Estevão Remígio de Freitas, 1145 - Bairro Monsenhor Otávio - Limoeiro do Norte, CE, Brasil - CEP: 62930-000

Email: bruna.yhang@ifce.edu.br

\section{Submissão:}

2 de maio de 2020

Aceito para publicação

9 de agosto de 2020

\section{RESUMO}

Introdução: A perda de massa muscular é uma consequência esperada do envelhecimento, porém o excesso de adiposidade também vem ascendendo nesta faixa etária. Ambos, isoladamente, comprometem a saúde e a qualidade de vida, mas impacto maior acontece quando se associam. Objetivo: Verificar a prevalência de risco de sarcopenia e obesidade sarcopênica entre idosos não-institucionalizados do município de Morada Nova-CE. Método: Estudo quantitativo, transversal, descritivo e analítico. Foi aplicado o Simple Questionnaire to Rapidly Diagnose Sarcopenia (SARC-F) e foram coletados peso, estatura, circunferência da panturrilha (CP) e dobras cutâneas tricipital (DCT), bicipital, subescapular e supra ilíaca, para diagnósticos de estado nutricional, sarcopenia e obesidade sarcopênica. Na estatística descritiva, calculou-se média e desvio-padrão. $\mathrm{Na}$ estatística inferencial, os testes de correlação de Pearson, ANOVA e qui-quadrado foram aplicados. Resultados: Participaram do estudo 121 idosos com idade maior ou igual a 60 anos, de ambos os sexos. A maioria dos idosos $(72 \%, \mathrm{n}=87)$ era do sexo feminino, com média de idade de $68,8 \pm 6,18$ anos, variando de 60 a 86 anos. Como principais achados, viu-se predomínio de eutrofia segundo o IMC, obesidade pela adequação de DCT e percentual de gordura (\%GC), ausência de depleção de massa muscular, sarcopenia e obesidade sarcopênica. Conclusões: A maioria das pessoas idosas não apresentou sarcopenia, nem obesidade sarcopênica. Não se encontrou associação entre estes dois diagnósticos, nem entre resultados da SARC-F e CP. As mulheres mostraram maior tendência para sarcopenia que os homens. Sugere-se a realização de estudos que possibilitem o estabelecimento de pontes de corte consensuais para o diagnóstico de sarcopenia e de obesidade sarcopênica, bem como trabalhos envolvendo a utilização do SARC-F para sua difusão e avaliação de sua sensibilidade para o diagnóstico de sarcopenia.

\section{ABSTRACT}

Introduction: The loss of muscle mass is an expected consequence of aging, but excess adiposity has also been increasing in this age group. Both in isolation compromise health and quality of life, but a greater impact happens when they associate. Objective: To verify the prevalence of risk for sarcopenia and sarcopenic obesity among elderly in the municipality of Morada Nova-CE. Methods: Quantitative, transversal, descriptive and analytical study. Simple Questionnaire to Rapidly Diagnosed Sarcopenia (SARC-F) was applied. After, weight, height, calf circumference (CP) and triceps skinfolds (DCT), bicipital, subscapular and supra iliac were collected for diagnoses of nutritional status, sarcopenia and sarcopenic obesity. In descriptive statistics, mean and standard deviation were calculated. In inferential statistics, Pearson's correlation tests, ANOVA and chisquare were applied. Results: A total of 121 elderly people aged 60 or over and of both sexes participated in the study. The majority of the elderly people $(72 \%, n=87)$ were female, with a mean age of $68.8 \pm 6.18$ years, ranging from 60 to 86 years. The main findings were: predominance of BMl eutrophy, obesity due to DCT adequacy and percentage of fat (\% GC), absence of muscle mass depletion, sarcopenia and sarcopenic obesity. Conclusions: Most elderly people did not have sarcopenia or sarcopenic obesity. No association was found between these two diagnoses, nor between the results of SARC-F and CP. Women were more likely to have sarcopenia than men. We suggest studies that allow the establishment of consensus bridges for the diagnosis of sarcopenia and sarcopenic obesity, as well as studies involving the use of SARC-F for its diffusion and evaluation of its sensitivity for the diagnosis of sarcopenia.

1. Nutricionista, Instituto Federal de Educação, Ciência e Tecnologia do Ceará, campus Limoeiro do Norte, Limoeiro do Norte, CE, Brasil.

2. Nutricionista, professora doutora, Instituto Federal de Educação, Ciência e Tecnologia do Ceará, campus Limoeiro do Norte, Limoeiro do Norte, CE, Brasil.

3. Graduanda em Nutrição, Instituto Federal de Educação, Ciência e Tecnologia do Ceará, campus Limoeiro do Norte, Limoeiro do Norte, CE, Brasil. 


\section{INTRODUÇÃO}

A relação entre alimentação, saúde e envelhecimento é encontrada desde o tempo dos papiros, no Antigo Egito, que continham várias recomendações envolvendo boa alimentação. Porém, apenas no século XIX o estudo do envelhecimento como ciência se expandiu. Em 1839, Carl Friedrich Canstatt contribuiu com pesquisas científicas que estimularam estudantes e leitores a refletirem sobre os assuntos que têm relação direta com a qualidade do envelhecimento. Com o passar dos anos esses estudos vêm evoluindo, em concomitância com o crescimento da população idosa e do interesse por uma parcela importante dos gestores de saúde de que esse público tenha qualidade de vida'.

O envelhecimento envolve mudanças fisiológicas diversas que reduzem a capacidade funcional e comprometem a qualidade de vida. Afeta funções corporais, principalmente músculo-esqueléticas, evidenciadas na forma de perda de massa muscular, com prejuízos na contração, movimento e locomoção². Portanto, a menor quantidade de massa muscular é uma consequência esperada com o passar dos anos. Contudo, quando a gordura corpórea passa a excessivamente substituir a massa livre de gordura, pode-se instalar um estado de sarcopenia ${ }^{3}$.

A definição recentemente proposta pelo European Working Group on Sarcopenia in Older People, em 2018, diz que a sarcopenia é um distúrbio progressivo e generalizado do músculo esquelético. $\bigcirc$ prejuízo da força muscular é o parâmetro diagnóstico primário, mas sua presença é confirmada quando a perda de massa muscular é detectada. A depleção de massa com a idade é de, aproximadamente, $1 \%$ a $2 \%$ por ano após os 50 anos. No entanto, a taxa de sarcopenia e suas repercussões variam segundo o estado de saúde, atividade física e dieta. Uma avaliação compatível com baixa força muscular associada com circunferência da panturrilha (CP) menor que $31 \mathrm{~cm}$ evidenciaria o diagnóstico da sarcopenia, se métodos mais precisos de avaliação da massa muscular não estiverem disponíveis ${ }^{4}$. A prevalência em diferentes grupos populacionais é variável, pois depende dos pontos de corte utilizados para sua definição e dos métodos de avaliação da força e da massa muscular, mas sabe-se que vem aumentando ao longo dos anos ${ }^{5}$.

Além do aumento da prevalência de sarcopenia na população idosa, crescem as taxas de obesidade, uma doença crônica de causa multifatorial, associada a fatores genéticos, fisiológicos e ambientais, como hábitos alimentares inadequados e inatividade física. Também pode ser definida como um excesso de gordura corporal, principalmente visceral ou abdominal ${ }^{3}$.

A condição de baixa massa livre de gordura (MLG) e elevada massa gorda (MG) é denominada obesidade sarcopênica, que resulta em piores funções físicas que a obesidade e a sarcopenia isoladas, pois a infiltração de gordura nos músculos impede a incorporação de aminoácidos e reduz a síntese protéica muscular ${ }^{6}$. Sua prevalência varia de acordo com os protocolos diagnósticos utilizados e a população estudada. Em estudos internacionais são encontrados valores que variam de $0,8 \%$ a $48 \% 5$. No Brasil, estudo atual encontrou prevalência de $6,5 \%$ a $9,4 \%$, dependendo do método de avaliação de gordura corporal utilizado 7 .

Dessa forma, diante das evidências de que sarcopenia e obesidade sarcopênica prejudicam saúde e qualidade de vida de idosos e em virtude desses diagnósticos estarem se tornando comuns nesta população, é importante que pesquisas com foco em suas triagens sejam realizadas, visando à prevenção e ao tratamento precoce. Assim, este estudo objetivou verificar a prevalência de risco para sarcopenia e obesidade sarcopênica entre idosos do município de Morada Nova - CE.

\section{MÉTODO}

Trata-se de um estudo quantitativo, descritivo e analítico realizado entre outubro de 2018 e janeiro de 2019, no Centro de Referência da Assistência Social (CRAS) da cidade de Morada Nova-CE. É quantitativo, pois todas as informações foram classificadas, analisadas e traduzidas em números. É descritivo e analítico porque, além de descrever, registrar e analisar a prevalência de risco de sarcopenia e obesidade, envolveu o entrecruzamento entre estas variáveis para se fechar um diagnóstico de obesidade sarcopênica. É ainda classificado quanto à fonte de dados como uma pesquisa de campo, pois o objeto de estudo foi investigado nas condições naturais em que os fenômenos ocorreram.

\section{Amostra}

A população do estudo foi constituída por pessoas idosas, isto é, com 60 anos ou mais, assistidas no referido CRAS. A amostra resultou em 169 participantes, considerando-se grau de confiança de 95\%, variância de 1,96 e erro amostral de $5 \%$.

Foram incluídos no estudo idosos de qualquer sexo. Os critérios de exclusão adotados foram: idosos com incapacidade de locomoção, desordem metabólica ou endócrina que afeta o sistema muscular ou sob uso de medicamentos que afetam diretamente a composição corporal.

Todos os voluntários assinaram um Termo de Consentimento Livre e Pós-esclarecido, contendo informações sobre os procedimentos, riscos e benefícios da pesquisa. $\bigcirc$ projeto foi avaliado e aprovado pelo Comitê de Ética em Pesquisa do Instituto Federal de Educação, Ciência e Tecnologia do Ceará (IFCE), sob parecer de número 2.891.062. 


\section{Instrumentos}

Foi aplicado o formulário de triagem de sarcopenia Simple Questionnaire to Rapidly Diagnose Sarcopenia (SARC-F), instrumento simples, de diagnóstico rápido, constituído por cinco componentes que analisam força, necessidade de assistência em caminhada, capacidade de levantar-se de uma cadeira, subir escadas e incidência de quedas ${ }^{8}$.

A massa corporal de todos os voluntários foi mensurada utilizando uma balança antropométrica tipo plataforma WELMY ${ }^{\circledR}$, graduada a cada 100 gramas, capacidade de 150 $\mathrm{kg}$ e precisão de 0,1 kg. A estatura foi mensurada utilizandose um estadiômetro portátil AVANUTRI ${ }^{\circ}$, com precisão de $0,1 \mathrm{~cm}$. Para a mensuração da massa corporal, os idosos estavam com roupas leves, sem sapatos e sem adereços, posicionados no centro da balança. Para a medida da estatura, os pés estavam juntos, com os calcanhares, nádegas e ombros encostados na barra escalonada do estadiômetro, olhando para a frente e com os braços estendidos para baixo.

A composição corporal foi determinada por meio da coleta das dobras cutâneas tricipital (DCT), bicipital, subescapular e supra ilíaca, utilizando um adipômetro CESCORF ${ }^{\circledR}$, que possui um sistema simplificado de leitura, base em ABS, material termoplástico rígido e leve e molas em aço zincado. As molas possuem pressão de $10 \mathrm{~g} / \mathrm{mm}^{2}$ e amplitude de leitura $80 \mathrm{~mm}$, com sensibilidade de $1 \mathrm{~mm}$, boa ergonomia manual, embalagem simples em acetato e amplitude de leitura de $80 \mathrm{~mm}$. A circunferência da panturrilha (CP) foi coletada com uma trena antropométrica $C E S C O R F \circledast$ segundo técnica validada?.

\section{Procedimentos}

Na aplicação do formulário SARC-F, a força foi avaliada perguntando-se sobre a dificuldade que os indivíduos tinham de levantar ou transportar $4,5 \mathrm{~kg}$. A assistência para caminhar foi avaliada questionando-se qual a dificuldade que eles tinham ao atravessar uma sala e se eles utilizavam auxílio ou precisavam de ajuda para fazer isso. Sobre a capacidade de levantar de uma cadeira, foi perguntado quanta dificuldade eles tinham de se transferir de uma cadeira ou cama e se eles precisavam de ajuda para fazer isso. A capacidade de subir escadas foi avaliada perguntando-se quanta dificuldade eles tinham ao subir um lance de 10 degraus. Por fim, foi avaliada a incidência de quedas. Todos os itens tinham como opções de resposta e respectivos escores atribuídos: $0=$ nenhuma dificuldade; 1 = alguma dificuldade e 2 = muita dificuldade, utiliza ajuda ou é incapaz de fazer sem ajuda8.

No SARC-F atribui-se 0 a 2 pontos para cada componente avaliado, dependendo do estado individual do idoso. Os escores globais da ferramenta variam de 0 a 10. Total de pontos inferior a quatro classifica o indivíduo como não sarcopênico, e maior ou igual a quatro como sarcopênico ${ }^{8}$.
Na avaliação antropométrica e de composição corporal, peso e estatura foram utilizados para o cálculo do índice de massa corporal (IMC), por meio do quociente peso/ estatura $^{2}$, sendo o peso corporal expresso em quilogramas $(\mathrm{kg})$ e a estatura em metros $(\mathrm{m})$. Os valores obtidos foram classificados de acordo com Lipschitz ${ }^{10}$, segundo os seguintes pontos de corte: $<22 \mathrm{~kg} / \mathrm{m}^{2}$, baixo peso; de 22 a $27 \mathrm{~kg} / \mathrm{m}^{2}$, adequação; $>27 \mathrm{~kg} / \mathrm{m}^{2}$, excesso de peso.

Foi utilizado o somatório das dobras cutânea bicipital, subescapular e supra ilíaca, conforme protocolo de Durnin e Wormersley ${ }^{11}$, para estimar o percentual de gordura (\%GC). Para classificar o estado nutricional, o \%GC foi classificado de acordo com Lohman'2. A análise dos valores de DCT baseou-se nos pontos de corte de Frisancho ${ }^{13}$. Para a CP foi adotada a classificação proposta pela Organização Mundial da Saúde? que indica redução de massa muscular quando seu valor é inferior a $31 \mathrm{~cm}$.

O diagnóstico de obesidade sarcopênica foi realizado mediante a associação de excesso de gordura corporal com depleção de CP. Considerou-se excesso de massa gorda $\%$ GC valor superior a $27 \%$ para mulheres e maior que $28 \%$ para homens ${ }^{14}$.

\section{Análise Estatística}

Os dados coletados foram inseridos em planilha do Microsoft Excel $\AA$, para posterior análise estatística no software Statistical Package for the Social Sciences (SPSS) ${ }^{\circledR}$, versão 20. Na estatística descritiva, estimou-se média, desvio-padrão, frequência absoluta e frequência percentual. Os idosos foram comparados por sexo em relação a todos os diagnósticos realizados. A relação de SARC-F com CP e obesidade sarcopênica foi investigada para o grupo no todo, sem separação por sexo.

O teste de correlação de Pearson foi utilizado para verificar a existência de correlação entre SARC-F e CP, enquanto variáveis contínuas. Com o teste ANOVA investigou-se a existência de associação entre tercis de SARC-F e CP. O teste qui-quadrado ou exato de Fisher foi utilizado para verificar a associação entre SARC-F e obesidade sarcopênica, enquanto variáveis categóricas. Para todos os testes adotou-se nível de significância de $5 \%$.

\section{RESULTADOS}

A amostra planejada para o estudo correspondeu a 169 pessoas idosas, contudo, participaram do estudo apenas 121, em virtude de reformas no estabelecimento de coleta de dados e férias coletivas dos profissionais. A maioria dos idosos $(72 \%, \mathrm{n}=87$ ) era do sexo feminino, com média de idade de 68,8 \pm 6,18 anos, variando de 60 a 86 anos.

A Tabela 1 apresenta as médias e desvios-padrão das variáveis antropométricas, de composição corporal e pontuação de SARC-F dos participantes da pesquisa. 
Tabela 1 - Médias e desvios-padrão das variáveis antropométricas, de composição corporal e pontuação de SARC-F dos idosos. Morada Nova-CE.

\begin{tabular}{lcccc}
\hline \multirow{2}{*}{ Variáveis } & Masculino & Feminino & Total & \\
\cline { 2 - 4 } & Média $\pm \mathrm{DP}$ & Média $\pm \mathrm{DP}$ & Média $\pm \mathrm{DP}$ & ${\text { Valor de } \boldsymbol{p}^{*}}^{*}$ \\
\hline Peso & $71,24 \pm 13,6$ & $63,89 \pm 13,07$ & $65,95 \pm 13,58$ & \\
IMC & $26,44 \pm 4,16$ & $27,5 \pm 5,14$ & $27,2 \pm 4,89$ & $\mathrm{p}=0,287$ \\
$\%$ DCT & $125,59 \pm 55,00$ & $91,42 \pm 29,21$ & $101,03 \pm 41,00$ & $\mathrm{p}=0,000$ \\
$\% G C$ & $28,60 \pm 6,86$ & $39,80 \pm 5,29$ & $36,65 \pm 7,6$ & $\mathrm{p}=0,000$ \\
SARC-F & $1,41 \pm 1,95$ & $2,82 \pm 2,29$ & $2,42 \pm 2,28$ & $\mathrm{p}=0,002$ \\
CP & $33 \pm 3,41$ & $33,09 \pm 3,51$ & $33,06 \pm 3,47$ & $\mathrm{p}=0,897$ \\
\hline
\end{tabular}

*Teste t de Student; $p<0,05$ como significante.

As médias de IMC e CP, por gênero e do grupo no todo, foram compatíveis, respectivamente, com eutrofia e ausência de depleção e não diferiram significativamente entre os sexos (IMC, $p=0,287 ; C P, p=0,897)$. As médias de adequação de DCT para o grupo e para os homens foram indicativos de sobrepeso, mas para o sexo feminino se enquadrou na faixa de desnutrição $e$, assim, foi significativamente menor que nos homens ( $p=$ 0,000). Os valores médios de \%GC para o grupo e por sexo sugeriram obesidade, sendo significativamente maior entre as mulheres $(p=0.000)$. Para o SARC-F, os resultados das médias abaixo de quatro demonstraram ausência de sarcopenia e foram significativamente maiores entre as mulheres $(p=0,002)$.

A Tabela 2 demonstra o comportamento dos tercis de CP em relação aos tercis de SARC-F.

Tabela 2 - Correlação entre tercis de SARC-F e CP entre os idosos. Morada Nova-CE, 2019.

\begin{tabular}{lcc}
\hline & \multicolumn{2}{c}{ SARC-F X CP } \\
\cline { 2 - 3 } & SARC-F & CP \\
\hline T1 & 0,268293 & 33,195 \\
T2 & 1,926829 & 32,951 \\
T3 & 5,25641 & 33,051 \\
\hline
\end{tabular}

* teste ANOVA, enquanto tercis ( $p=0,864)$; **teste de correlação de Pearson, enquanto variáveis contínuas ( $r=0,038, p=0,677)$.

Ao comparar os tercis 1 e 3, verificou-se uma correlação inversa, mas fraca, e não significativa estaticamente $(p=$ $0,864)$ entre as variáveis. Ao analisar a correlação entre CP e SARC-F enquanto variáveis contínuas, também se detectou correlação inversa muito fraca entre estas variáveis, sem significância estatística $(r=-0,038, p=0,677)$.

A Tabela 3 mostra a distribuição do grupo de idosos de acordo com o diagnóstico de sarcopenia segundo a SARC-F, bem como de obesidade sarcopênica.
Tabela 3 - Categorização nutricional dos idosos, por gênero, segundo diagnóstico antropométrico, de composição corporal e triagem de sarcopenia e obesidade sarcopênica. Morada Nova-CE, 2019.

\begin{tabular}{lcccc}
\hline & \multicolumn{2}{c}{ SARC-F } & \multicolumn{2}{c}{ Obesidade Sarcopênica } \\
\cline { 2 - 5 } & $\mathbf{n}$ & $\%$ & $\mathbf{n}$ & $\%$ \\
\hline Sim & 23 & 19,00 & 21 & 17,00 \\
Não & 98 & 81,00 & 100 & 83,00 \\
Total & 121 & 100 & 121 & 100 \\
\hline
\end{tabular}

*Teste qui-quadrado ou exato de Fisher, enquanto variáveis categóricas $(\mathrm{p}=0,537)$

Predominou entre os idosos a ausência de sarcopenia e de obesidade sarcopênica. Contudo, não foi detectada associação significante entre triagem de sarcopenia segundo a SARC-F e diagnóstico de obesidade sarcopênica $(p=0,537)$.

A Tabela 4 apresenta a distribuição do grupo, em geral e por gênero, segundo os diagnósticos nutricionais a partir das variáveis antropométricas, de composição corporal, sarcopenia e obesidade sarcopênica.

Houve predomínio no grupo de eutrofia segundo o IMC, mas também um quantitativo importante de sobrepeso. Também, foi observada obesidade pela adequação de DCT, obesidade segundo o\%GC, ausência de depleção de massa e de sarcopenia, respectivamente, conforme CP e SARC-F, e ausência de obesidade sarcopênica. Para o IMC, predominou entre os homens a eutrofia e, entre as mulheres, sobrepeso. Para adequação de DCT prevaleceu para homens a obesidade, já para as mulheres, a desnutrição grave. E para o $\%$ GC, em ambos os gêneros, predominou a obesidade.

\section{DISCUSSÃO}

Este estudo buscou investigar a prevalência de risco de sarcopenia e de obesidade sarcopênica entre pessoas idosas não-institucionalizadas, residentes no município de Morada 
Tabela 4 - Categorização nutricional dos idosos segundo variáveis antropométricas, de composição corporal e triagem de sarcopenia e obesidade sarcopênica, Morada Nova-CE, 2019.

\begin{tabular}{|c|c|c|c|c|c|c|c|}
\hline \multirow{2}{*}{ Variáveis } & \multirow{2}{*}{ Diagnóstico } & \multicolumn{2}{|c|}{ Masculino } & \multicolumn{2}{|c|}{ Feminino } & \multicolumn{2}{|c|}{ Total } \\
\hline & & $\mathrm{n}$ & $\%$ & $\mathrm{n}$ & $\%$ & $\mathrm{n}$ & $\%$ \\
\hline \multirow{4}{*}{ IMC } & Magreza & 5 & 15,00 & 14 & 16,00 & 19 & 16,00 \\
\hline & Eutrofia & 18 & 53,00 & 36 & 41,00 & 54 & 44,00 \\
\hline & Sobrepeso & 11 & 32,00 & 37 & 43,00 & 48 & 40,00 \\
\hline & Total & & & & & 121 & 100 \\
\hline \multirow{7}{*}{ Adequação da DCT } & Desnutrição grave & 5 & 15,00 & 21 & 24,00 & 26 & 22,00 \\
\hline & Desnutrição moderada & - & - & 5 & 6,00 & 5 & 4,00 \\
\hline & Desnutrição leve & 1 & 3,00 & 17 & 20,00 & 18 & 15,00 \\
\hline & Eutrofia & 9 & 26,00 & 19 & 22,00 & 28 & 23,00 \\
\hline & Sobrepeso & 1 & 3,00 & 10 & 11,00 & 11 & 9,00 \\
\hline & Obesidade & 18 & 53,00 & 15 & 17,00 & 33 & 27,00 \\
\hline & Total & & & & & 121 & 100 \\
\hline \multirow{5}{*}{$\% G C$} & Normal & 2 & 6,00 & 0 & 0 & 2 & 2,00 \\
\hline & Média & 1 & 3,00 & 0 & 0 & 1 & 1,00 \\
\hline & Acima da média & 8 & 23,00 & 5 & 6,00 & 13 & 10,00 \\
\hline & Obesidade & 23 & 68,00 & 82 & 94,00 & 105 & 87,00 \\
\hline & Total & & & & & 121 & 100 \\
\hline \multirow{3}{*}{$\mathrm{CP}$} & Depleção & 9 & 26,00 & 21 & 25,00 & 30 & 25,00 \\
\hline & Não depleção & 25 & 74,00 & 66 & 75,00 & 91 & 75,00 \\
\hline & Total & & & & & 121 & 100 \\
\hline \multirow{3}{*}{ SARC-F } & Sarcopênico & 2 & 6,00 & 21 & 23,00 & 23 & 19,00 \\
\hline & Não sarcopênico & 32 & 94,00 & 66 & 75,00 & 98 & 81,00 \\
\hline & Total & & & & & 121 & 100 \\
\hline \multirow[t]{3}{*}{ Obesidade Sarcopênica } & $\operatorname{Sim}$ & 3 & 9,00 & 18 & 21,00 & 21 & 17,00 \\
\hline & Não & 31 & 91,00 & 69 & 79,00 & 100 & 83,00 \\
\hline & Total & & & & & 121 & 100 \\
\hline
\end{tabular}

Nova, interior do Ceará. Como principais achados, destacase que foi baixa a prevalência de sarcopenia e de obesidade sarcopênica no grupo e que não houve associação entre estes dois diagnósticos. A adequação de DCT foi significativamente menor no público feminino, mas \%GC e SARC-F foram significativamente maiores neste gênero.

Foi possível constatar, quanto ao IMC, que o grupo está predominantemente eutrófico, mas alto percentual tem sobrepeso. Quando se analisa adequação de DCT, encontra-se predomínio de obesidade entre homens e desnutrição grave entre as mulheres. $\bigcirc \% G C$ mostra obesidade em ambos os gêneros. Ao triar sarcopenia através do questionário SARC-F, a maior parte do grupo não é sarcopênica, mas o diagnóstico de sarcopenia é maior no sexo feminino. A medida da CP, sensível para avaliação de massa muscular, que é componente da triagem de sarcopenia, mostrou, na maioria dos idosos, ausência de depleção. A ausência de obesidade sarcopênica também predominou entre os idosos. Portanto, 
sarcopenia e obesidade sarcopênica não representam uma problemática neste grupo. Contudo, medidas para prevenilas devem ser adotadas, visto que a perda de massa muscular é uma alteração fisiológica do envelhecimento, podendo resultar em sarcopenia, assim como em obesidade sarcopênica devido ao diagnóstico de obesidade ou sobrepeso nos parâmetros IMC, DCT e \%GC ${ }^{3}$.

De acordo com a média de IMC, a população se encontra em estado de eutrofia, entretanto, essa variável não possibilita determinar que parte do peso é composta especificamente por massa gorda e por massa magra. Assim, uma vez que o idoso passa por diversas alterações fisiológicas, deve-se utilizar outras medidas antropométricas para avaliação do estado nutricional deste público.

Os resultados demonstraram que os idosos avaliados apresentam IMC dentro da faixa de eutrofia ${ }^{10}$ para idosos (26,44 kg/m² para homens e $27,5 \mathrm{~kg} / \mathrm{m}^{2}$ para mulheres), sendo o valor encontrado semelhante ao apresentado por Baumgartner ${ }^{14}$ para 0 sexo feminino e superior ao evidenciado pelo sexo masculino $\left(24,4 \mathrm{~kg} / \mathrm{m}^{2}\right.$ em homens e 27,1 $\mathrm{kg} / \mathrm{m}^{2}$ em mulheres). De acordo com o intervalo de classificação do estado nutricional de idosos pelo IMC, estabelecido por Lipschitz ${ }^{10}$, o diagnóstico é de eutrofia, porém, quando confrontados com o valor ideal para a idade (22 a $27 \mathrm{~kg} / \mathrm{m}^{2}$ ), é possível detectar que a faixa de referência é ultrapassada nas mulheres e está próximo ao limite superior nos homens.

Com relação à dobra cutânea tricipital, existem padrões de referência específicos para idosos, propostos por Frisancho ${ }^{11}$. No estudo de Machado et al. ${ }^{15}$, observou-se elevada diferença entre as médias de DCT entre os gêneros, pois a média do valor entre as mulheres correspondeu quase ao dobro do encontrado entre os homens, achado contrário ao do presente estudo, em que a diferença entre os sexos foi grande, mas com valor bem maior para os homens, compatível com obesidade. Já no estudo de Sperotto e Spinelliib, a maioria dos idosos apresentou desnutrição (80\%) em relação a esse parâmetro, enquanto apenas $5 \%$, obesidade. Ressaltase que a precisão da medida da DCT pode ser influenciada pela diminuição da elasticidade e hidratação da pele, relacionada à idade, bem como pela redistribuição do tecido adiposo no idoso. Entretanto, seu uso é recomendado por estudiosos por ser não invasivo, de baixo custo e aplicável em estudos de campo. Apesar de divergências na literatura sobre o uso de dobras cutâneas em idosos, a mais utilizada é a tricipital ${ }^{15}$.

Em relação ao \%GC, estudo de Munaretto et al. ${ }^{17}$ demonstrou que o excesso de peso é maior nos homens, sendo o seu valor médio de $33 \%$ neste gênero, resultado contrário ao presente estudo, no qual o grupo masculino teve uma média de $28,6 \%$, bem menor que entre as mulheres. Neste mesmo estudo, a prevalência de magreza foi maior entre as mulheres $(5,25 \%)$, dado também contrário ao desse estudo, com maior grau de obesidade para o grupo feminino. De acordo com dados do presente estudo, o \%GC, para ambos os grupos, foi indicativo de obesidade, estando em concordância com o perfil de transição nutricional que vem ocorrendo ao longo dos anos, em que o excesso de peso só cresce entre os idosos ${ }^{3}$.

Farooq et al. ${ }^{18}$ encontraram em seu estudo um número maior de mulheres com alto percentual de gordura corporal (mulheres: $43,4 \pm 6,3 \%$ versus homens: $32,7 \pm 8,8 \%$ ), o mesmo evidenciado por Gomes et al. ${ }^{19}$ entre mulheres com média de idade de 80 anos, em que $43 \%$ foram identificadas com obesidade, comparadas a $32 \%$ dos homens.

Com relação à sarcopenia, esta não foi percebida na maior parte do grupo, porém a análise por gênero sugere para a triagem pela SARC-F e para o diagnóstico de obesidade sarcopênica uma possível tendência ou vulnerabilidade maior para as mulheres, que tiveram maior percentual de resultado positivo para as triagens. Além disso, na adequação da DCT, enquanto o gênero masculino apresenta predomínio de obesidade, no gênero feminino, houve destaque para a desnutrição grave, que geralmente está associada com perda de massa muscular. Quando comparadas aos homens ${ }^{20}$, as mulheres parecem ter uma redução de função e de massa magra mais significante com a idade e, portanto, apresentam maior risco de acometimento por sarcopenia, sendo que as mulheres idosas, por já terem passado pela menopausa, sofrem alterações hormonais, especialmente a diminuição do estrógeno, o que conduz a um aumento da massa gorda, declínio da massa magra e de massa óssea.

A triagem de sarcopenia pelo questionário SARC-F (81\% não sarcopênico e 19\% sarcopênico) mostrou resultados próximos ao do estudo de Santos et al. ${ }^{21}$, realizado com 116 idosos longevos entre 80 e 95 anos, em que 87,4\% não apresentaram sarcopenia.

Acerca da CP, apenas $1 / 4$ dos participantes foi diagnosticado com depleção. Semelhante a este estudo, Paula et al. ${ }^{22}$ concluíram que a aferição da CP não identificou sarcopenia na maioria das idosas participantes do estudo. Tem sido evidenciado, atualmente, que o ponto de corte de 31 $\mathrm{cm}$, amplamente utilizado, inclusive na presente pesquisa, apresenta baixa sensibilidade para a detecção de depleção muscular ${ }^{23}$. Caso isto venha a se confirmar, a prejuízo de massa magra pode ter maior prevalência neste grupo.

A ressonância magnética e a tomografia computadorizada são os métodos avaliativos padrão-ouro para mensuração da quantidade de massa muscular, entretanto, a CP foi proposta por pesquisadores e especialistas da área como alternativa a estes referidos, caso não estejam disponíveis ${ }^{4}$. A OMS também considera a CP uma medida sensível da massa muscular nos idosos, capaz de indicar alterações 
que ocorrem com o envelhecimento e com a redução de atividade física. Além disso, há evidência de associação entre $\mathrm{CP}$ inferior a $31 \mathrm{~cm}$ e incapacidade, assim como de valores adequados de CP com menor nível de fragilidade e melhor desempenho funcional ${ }^{9}$.

Sobre a obesidade sarcopênica, no estudo de Santos et al. ${ }^{21}$, encontrou-se valores aproximados (83\% não obeso sarcopênico e $17 \%$ obeso sarcopênico) aos do presente estudo (84,6\% sem obesidade sarcopênica e $15,4 \%$ com diagnóstico). Já Oliveira et al. ${ }^{7}$, ao investigarem o desfecho de forma completamente objetiva, a qual incluiv a avaliação da força muscular, detectaram prevalências menores, de $6,5 \%$ quando a adiposidade foi diagnosticada por meio de bioimpedância elétrica e 9,4\% pela circunferência da cintura $^{7}$. Independentemente dessas taxas, a triagem de obesidade sarcopênica deve ser conduta contínua no público idoso, pois representa um risco à saúde e qualidade de vida, principalmente no grupo aqui estudado, em que houve maior prevalência de pessoas com \%GC e DCT indicativos de obesidade.

Tem-se evidência de que a utilização do SARC-F de forma isolada é insuficiente para rastreamento de sarcopenia, então, para uma investigação diagnóstica mais precisa, deve-se associar a ferramenta com outras medidas, como circunferência da panturrilha, conforme realizado neste estudo e em trabalho de Barbosa-Silva et al. ${ }^{24}$. Apesar de estatisticamente não ter sido significante, percebeu-se uma fraca correlação inversa entre os tercis 1 e 3 de SARC-F e $\mathrm{CP}$, que sugere maior risco de sarcopenia diante da depleção de CP. No estudo de lto e Kanno ${ }^{25}$, foram identificados como sarcopênicos $23 \%$ pelo SARC-F e $30 \%$ pelo SARC-F + CP, demonstrando que a associação dessas ferramentas apresenta benefícios na identificação de idosos com fragilidade.

De acordo com Cruz-Jentoft et al. ${ }^{4}$, a infiltração de gordura no tecido muscular esquelético interfere na sua função e geração de força, reduzindo a performance e a qualidade de vida desses idosos. A adoção de um estilo de vida ativo, seguindo um programa de treinamento físico adequado ${ }^{2}$, especialmente de resistência ${ }^{3}$, pode pelo menos parcialmente melhorar esta composição corporal ${ }^{2,3}$. Também, restrição calórica de 200 a $500 \mathrm{kcal} / \mathrm{dia}$, combinada com ingestão de proteínas de 1 a $1,5 \mathrm{~g} / \mathrm{kg}$, é fundamental para prevenir o catabolismo muscular em idosos com obesidade ou com risco de obesidade sarcopênica ${ }^{3}$. Estas estratégias, portanto, precisam ser levadas em consideração na assistência à saúde prestada às pessoas idosas que participaram deste estudo.

Apesar de ter possibilitado vários achados, identificou-se alguns empecilhos ao longo deste estudo que podem representar limitações, como a falta de consenso na literatura para pontos de corte sugestivos do diagnóstico de sarcopenia e de obesidade sarcopênica, quantidade restrita de estudos acerca da temática, bem como de pesquisas nacionais que utilizaram a ferramenta SARC-F, limitando comparações. Portanto, seria importante a realização de mais estudos utilizando este instrumento em confronto com o diagnóstico objetivo de sarcopenia, não só com o intuito de difundi-la, mas também de melhor avaliar sua fidedignidade, uma vez que esta ferramenta é simples, de fácil aplicação e pode ter grande utilidade em nível de saúde pública, possibilitando diagnóstico precoce de sarcopenia e intervenção ágil. Há a necessidade ainda de estudos de rigor metodológico que viabilizem o estabelecimento de pontos de corte consensuais para sarcopenia e obesidade sarcopênica.

\section{CONCLUSÃO}

Conclui-se que a maioria das pessoas idosas estudadas não apresentou sarcopenia, nem obesidade sarcopênica, mas o grupo feminino mostrou maior tendência para sarcopenia que os homens. Não se encontrou associação entre estes dois diagnósticos, nem entre resultados da SARC-F e CP.

Entre as mulheres, predominou a eutrofia para IMC, desnutrição para adequação de DCT, obesidade para \%GC e ausência de depleção de massa muscular segundo a CP. Já entre os homens, predominou também a eutrofia para IMC, obesidade para DCT, obesidade para \%GC e ausência de depleção segundo CP.

É necessário que este grupo de idosos tenha acesso a uma assistência à saúde que possibilite melhoria do hábito alimentar e incentive a prática de atividade física, de modo a prevenir obesidade, sarcopenia e obesidade sarcopênica. Há necessidade do estabelecimento de pontos de corte consensuais para o diagnóstico das duas últimas e, para isso, desenvolvimento de mais estudos acerca da temática.

Em virtude da praticidade do instrumento SARC-F, necessita-se da ampliação de pesquisas que possibilitem sua difusão e avaliação de sua sensibilidade para o diagnóstico de sarcopenia, uma vez que essa ferramenta em nível de saúde pública possibilitaria diagnóstico precoce de sarcopenia para intervenção.

\section{REFERÊNCIAS}

1. Silva MLN, Marucci MFN, Roediger MA. Tratado de nutrição em gerontologia. Barueri: Manole; 2014.

2. Frontera WR. Physiologic changes of the musculoskeletal system with aging: a brief review. Phys Med Rehabil Clin N Am. 2017;28(4):705-11.

3. McKee A, Morley JE. Obesity in the elderly [Internet]. Bethesda; 2018 [citado 2020 abr. 14]. Disponível em: https://www.ncbi. nlm.nih.gov/books/NBK532533/

4. Cruz-JentoftAJ,BahatG, BauerJ,BoirieY,BruyèreO, Cederholm $\mathrm{T}$, et al; Writing Group for the European Working Group on Sarcopenia in Older People 2 (EWGSOP2), and the Extended Group for EWGSOP2. Sarcopenia: revised European consensus on definition and diagnosis. Age Ageing. 2019;48(1):16-31. 
5. Batsis JA, Villareal DT. Sarcopenic obesity in older adults: aetiology, epidemiology and treatment strategies. Nat Rev Endocrinol. 2018;14(9):513-37.

6. Parr EB, Coffey VG, Hawley JA. 'Sarcobesity': a metabolic conundrum. Maturitas. 2013;74(2):109-13.

7. Oliveira TM, Roriz AKC, Barreto-Medeiros JM, Ferreira AJF, Ramos L. Sarcopenic obesity in community-dwelling older women, determined by different diagnostic methods. Nutr Hosp. 2019;36(6):1267-72.

8. Malmstrom TK, Morley JE. SARC-F: a simple questionnaire to rapidly diagnose sarcopenia. J Am Med Dir Assoc. 2013;14(8):531-2.

9. World Health Organization. Physical status: the use and interpretation of anthropometry. Report of a WHO Expert Committee. World Health Organ Tech Rep Ser. 1995;854:1-452.

10. Lipschitz DA. Screening for nutritional status in the elderly. Prim Care. 1994;21(1):55-67.

11. Durnin JV, Womersley J. Body fat assessed from the total body density and its estimation from skinfold thickness: measurements on 481 men and women aged from 16 to 72 years. Br J Nutr. 1974;32(1):77-97.

12. Lohman G. Body fat measurement goes high-tech: not all are created equal. ACSMS Health Fit. 1997;1(1):30-5.

13. Frisancho AR. Anthropometric standards for the assessment of growth and nutritional status. Ann Arbor: University of Michigan Press; 1990.

14. Baumgartner RN. Body composition in healthy aging. Ann N Y Acad Sci. 2000;904:437-48.

15. Machado RSP, Coelho MASC, Coelho KSC. Percentual de gordura corporal em idosos: comparação entre os métodos de estimativa pela área adiposa do braço, pela dobra cutânea tricipital e por bioimpedância tetrapolar. Rev Bras Geriatr Gerontol. 2010;13(1):17-27.

16. Sperotto FM, Spinelli RB. Avaliação nutricional em idosos independentes de uma instituição de longa permanência no município de Erechim-RS. Perspectiva. 2010;34(125):105-16.
17. Munaretto L, Baratto I, Gatti RR, Frutuoso T. Body mass index and intake of macronutrients in the elderly. RBONE. 2014;8(46):115-21.

18. Farooq A, Knez WL, Knez K, Al-Noaimi A, Grantham J, Mohamed-Ali V. Gender differences in fat distribution and inflammatory markers among Arabs. Mediators Inflamm. 2013;2013:497324.

19. Gomes IC, Santos VR, Christofaro DG, Santos LL, Freitas Júnior IF. The most frequent cardiovascular risk factors in Brazilian aged 80 years or older. J Appl Gerontol. 2013;32(4):408-21.

20. Du Y, Wang X, Xie H, Zheng S, Wu X, Zhu X, et al. Sex differences in the prevalence and adverse outcomes of sarcopenia and sarcopenic obesity in community dwelling elderly in East China using the AWGS criteria. BMC Endocr Disord. 2019;19(1):109.

21. Santos VRD, Gomes IC, Bueno DR, Christofaro DGD, Freitas IF Jr, Gobbo LA. Obesity, sarcopenia, sarcopenic obesity and reduced mobility in Brazilian older people aged 80 years and over. Einstein (Sao Paulo). 2017;15(4):435-40.

22. Paula JA, Wamser EL, Gomes ARS, Valderramas SR, Cardoso Neto J, Schieferdecker MEM. Análise de métodos para detectar sarcopenia em idosas independentes da comunidade. Rev Bras Geriatr Gerontol. 2016;19(2):235-46.

23. Pagotto V, Santos KFD, Malaquias SG, Bachion MM, Silveira EA. Calf circumference: clinical validation for evaluation of muscle mass in the elderly. Rev Bras Enferm. 2018;71(2):322-8.

24. Barbosa-Silva TG, Menezes AM, Bielemann RM, Malmstrom TK, Gonzalez MC; Grupo de Estudos em Composição Corporal e Nutrição (COCONUT). Enhancing SARC-F: improving sarcopenia screening in the clinical practice. J Am Med Dir Assoc. 2016;17(12):1136-41.

25. Ito C, Kanno P. Prevalência de pacientes sarcopênicos através do SARC-F associado CP e sua capacidade de identificar pacientes com força de preensão palmar reduzida num ambulatório de Geriatria. Int J Nutrol. 2018;1(11):1-2.

Local de realização do estudo: Instituto Federal de Educação, Ciência e Tecnologia do Ceará, campus Limoeiro do Norte, Limoeiro do Norte, CE, Brasil.

Conflito de interesse: Os autores declaram não haver. 